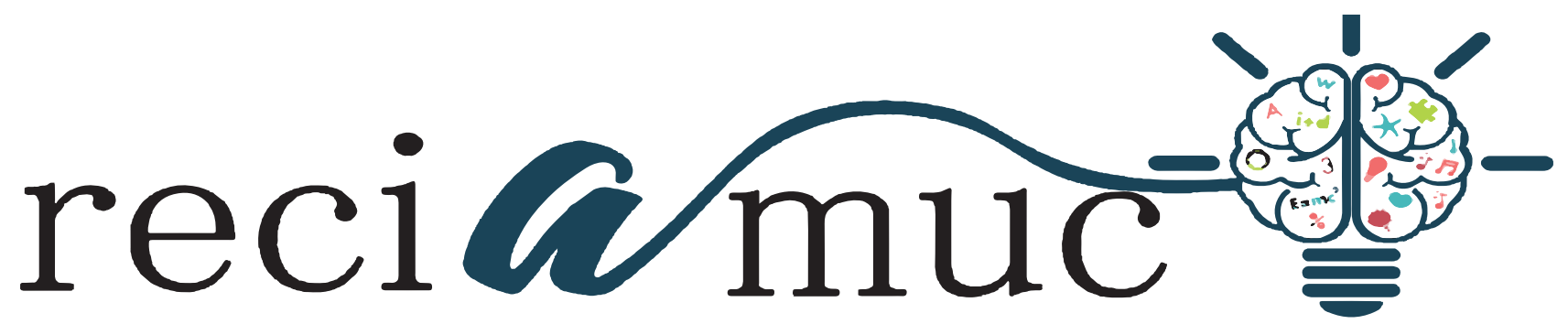

DOI: $10.26820 /$ reciamuc/4.(1).enero.2020.267-275

URL: https://reciamuc.com/index.php/RECIAMUC/article/view/432

EDITORIAL: Saberes del Conocimiento

REVISTA: RECIAMUC

ISSN: $2588-0748$

TIPO DE INVESTIGACIÓN: Artículo de Revisión CÓDIGO UNESCO: 3213.13 Estomatología

PAGINAS: $267-275$

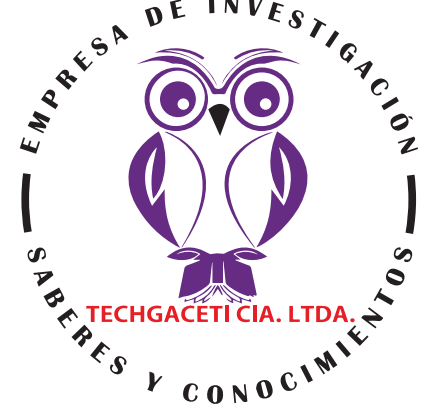

\title{
Hombro Congelado. Diagnóstico y tratamiento
}

\author{
Frozen Shoulder. \\ Diagnosis and treatment \\ Ombro Congelado. \\ Diagnóstico e tratamento
}

Kevin Wilfrido Versoza Castro'; Carla Anabell Zambrano Sanchez2; Lissette Katherine Masache Galvez ${ }^{3}$; Byron Mauricio Sanchez Ortiz ${ }^{4}$

RECIBIDO: 18/11/2019 ACEPTADO: 20/12/2019 PUBLICADO: 31/01/2020

1. Médico; Investigador Independiente; Guayaquil, Ecuador; versozakevin@gmail.com; (D) https://orcid.org/00000001-6458-5261

2. Médico; Investigador Independiente; Guayaquil, Ecuador; karla_zambrano_sanchez@hotmail.com; (DD https:// orcid.org/0000-0001-6083-1285

3. Médico; Investigador Independiente; Universidad de Guayaquil; Guayaquil, Ecuador; liscat_5@hotmail.com; (D) https://orcid.org/0000-0003-2163-5112

4. Médico; Investigador Independiente; Universidad de Guayaquil; Guayaquil, Ecuador; byron_sanchez_16@ hotmail.com; (D) https://orcid.org/0000-0003-1676-2328

\section{CORRESPONDENCIA \\ Kevin Wilfrido Versoza Castro \\ versozakevin@gmail.com \\ Guayaquil, Ecuador}




\section{RESUMEN}

A lo largo de la historia, la Capsulitis Adhesiva también conocida como hombro congelado, ha sido vista como una patología de causa desconocida, curso variable, poco predecible y de tratamiento controversial. Es una dolencia que se presenta en esta importante articulación ocasionando dolor en la región superior del tronco y que genera la pérdida progresiva de la movilidad pasiva del hombro. El presente trabajo, concebido en el marco de una metodología de revisión, se enfoca en referir algunos criterios recientes o vigentes de distintos autores en cuanto al diagnostico y tratamiento del hombro congelado. El tiempo de evolución de la enfermedad varía de caso a caso, pero es muy común que el hombro congelado interfiera en las actividades normales de la vida del paciente durante al menos 2 años. Algunos pacientes pueden permanecer con secuelas, perdiendo definitivamente cerca de un 15\% de la movilidad del hombro. El tratamiento inicial, sin importar la etiología, es siempre conservador, con terapia física, ejercicios de elongación, antiinflamatorios no esteroides e infiltraciones con corticoides. La opción quirúrgica, se considera a las 6 a 12 semanas de un tratamiento conservador fallido, siendo la técnica quirúrgica más utilizada la liberación capsular artroscópica. En este sentido es importante la valoración del especialista, los conocimientos en esta patología para su inmediata revisión realizando exámenes de rutina dando al paciente a tratar un diagnostico oportuno y colocando el tratamiento a seguir.

Palabras clave: Hombro congelado, Capsulitis adhesiva, Movilidad, Articulación, Terapia.

\section{ABSTRACT}

Throughout history, Adhesive Capsulitis also known as frozen shoulder, has been seen as a pathology of unknown cause, variable course, little predictable and controversial treatment. It is a condition that occurs in this important joint causing pain in the upper region of the trunk and that generates the progressive loss of passive mobility of the shoulder. The present work, conceived in the framework of a review methodology, focuses on referring some recent or current criteria of different authors regarding the diagnosis and treatment of frozen shoulder. The time of evolution of the disease varies from case to case, but it is very common for the frozen shoulder to interfere with the normal activities of the patient's life for at least 2 years. Some patients may remain with sequelae, definitely losing about $15 \%$ of shoulder mobility. The initial treatment, regardless of the etiology, is always conservative, with physical therapy, elongation exercises, nonsteroidal anti-inflammatory drugs and corticosteroid infiltration. The surgical option is considered at 6 to 12 weeks of a failed conservative treatment, the most commonly used surgical technique being arthroscopic capsular release. In this sense it is important to assess the specialist, knowledge in this pathology for immediate review by performing routine exams giving the patient to treat a timely diagnosis and placing the treatment to follow.

Keywords: Frozen shoulder, Adhesive capsulitis, Mobility, Joint, Therapy.

\section{RESUMO}

Ao longo da história, a capsulite adesiva, também conhecida como ombro congelado, tem sido vista como uma patologia de causa desconhecida, curso variável, pouco tratamento previsível e controverso. É uma condição que ocorre nessa importante articulação, causando dor na região superior do tronco e que gera a perda progressiva da mobilidade passiva do ombro. O presente trabalho, concebido no âmbito de uma metodologia de revisão, enfoca a referência a alguns critérios recentes ou atuais de diferentes autores quanto ao diagnóstico e tratamento do ombro congelado. O tempo de evolução da doença varia de caso para caso, mas é muito comum o ombro congelado interferir nas atividades normais da vida do paciente por pelo menos 2 anos. Alguns pacientes podem permanecer com sequelas, perdendo definitivamente cerca de $15 \%$ da mobilidade do ombro. O tratamento inicial, independentemente da etiologia, é sempre conservador, com fisioterapia, exercícios de alongamento, anti-inflamatórios não esteróides e infiltração de corticosteróides. A opção cirúrgica é considerada em 6 a 12 semanas de falha do tratamento conservador, sendo a técnica cirúrgica mais comum a liberação capsular artroscópica. Nesse sentido, é importante avaliar o especialista, conhecimento nessa patologia, para revisão imediata, realizando exames de rotina, dando ao paciente o tratamento oportuno e colocando o tratamento a seguir.

Palavras-chave: Ombro congelado, Capsulite adesiva, Mobilidade, Articulações, Terapia. 


\section{Introducción}

A lo largo de la historia, la Capsulitis Adhesiva ha sido vista como una patología de causa desconocida, curso variable, poco predecible y de tratamiento controversial. También conocida como (hombro congelado). "Se define como la pérdida progresiva de la movilidad pasiva del hombro y se acompaña de dolor difuso que predomina en la región anterolateral del hombro". (Serrano \& Abush, Capsulitis adhesiva, 2017)

"Los tejidos alrededor de la articulación se vuelven rígidos, se forma tejido cicatricial, y mover el hombro se vuelve difícil y doloroso. La afección suele aparecer lentamente, y luego desaparece lentamente en el transcurso de un año o más tiempo". (Healthwise, 2019).

En estudio realizado por Ramos (2017) se evidencia que:

La Capsulitis adhesiva tiene una incidencia de 3 a 5\% en la población general. La prevalencia de hombro doloroso se estima entre el 16 y 26\%. Constituye la tercera causa más frecuente de consulta entre las afecciones del sistema osteomioarticular en la atención primaria. Además anualmente el $1 \%$ de los adultos acude a consulta por dolencias en el hombro.

Se conocen 2 tipologías diferenciadas de hombro congelado de acuerdo a Ortiz, Et. Al (2010):

- Hombro congelado primario. En aquellos pacientes en los que no se encuentra ningún factor que incite la aparición de la patología y que no haya ninguna anormalidad en la observación.

- Hombro congelado secundario. Incluye casos de rigidez post traumática, hombro congelado asociado con DM, dolor por infarto post miocardial, y desordenes inflamatorios entre otros.

En esta revisión se abordarán las definiciones históricas, la sintomatología y clasifica- ción de la capsulitis por etapas, métodos de diagnostico y diferentes modalidades de tratamiento, con el objetivo de dar a conocer esta patología y describir el abordaje adecuado para la valoración y manejo de los pacientes con rigidez de hombro. Con fines prácticos, se usarán de forma indistinta los términos "capsulitis adhesiva" y "hombro congelado".

\section{Materiales y Métodos}

El presente trabajo, concebido en el marco de una metodología de revisión, se enfoca en referir algunos criterios recientes o vigentes de distintos autores en cuanto al diagnostico y tratamiento del hombro congelado.

Entre las bases de datos consultadas resaltaron la de: Biblioteca Virtual de la Salud (BVS), SciELO, MedlinePlus, Dialnet, Mayoclinic, entre otras. Como términos de búsqueda se utilizaron las expresiones "hombro congelado" y "capsulitis adhesiva", a los que seguidamente se le aplicaron criterios de selección tales como: idioma español (e inglés en algunos casos); publicación entre 2009 y 2019 (ambos inclusive), salvo algunas excepciones; acceso completo y abierto; en materia de salud y medicina; estudios referidos a humanos; tipo de bibliografía, artículos científicos, estudios o reportes de casos, consensos, protocolos, tesis de grado, posgrado y doctorado, noticias científicas, boletines y/o folletos de instituciones oficiales o privadas de reconocida trayectoria en el área de la salud, medicina o científico académica, y demás, monografías y otros documentos que, a criterio propio, mostraran información de interés en base a la observación de la evidencia científica referida en sus contenidos.

Se prosiguió con la lectura crítica y análisis interpretativo de todo el contenido seleccionado al que se le trato como evidencia, resultando todo este proceso en la escogencia de quince (15) fuentes bibliográficas que fundamentan el criterio aquí expuesto. 


\section{Resultados}

El hombro es un conjunto de varias articulaciones que se combinan con tendones y músculos para permitir un amplio rango de movimiento en el brazo. Ugalde, Carlos, Et. Al (2013) lo definen como:

Una estructura compleja conformada por la parte proximal del húmero, la clavícula, la escápula, y las uniones de estos huesos con el esternón, la caja torácica, y tejidos blandos. Está constituido por varias articulaciones: esternoclavicular, acromioclavicular, glenohumeral y escapulotorácica, las cuales trabajan juntas a un ritmo sincrónico, para permitir el movimiento. Esta complejidad le confiere la característica de ser una de las articulaciones más móviles del cuerpo, por lo tanto un sitio de múltiples lesiones y patologías inflamatorias, traumáticas, así como degenerativas.

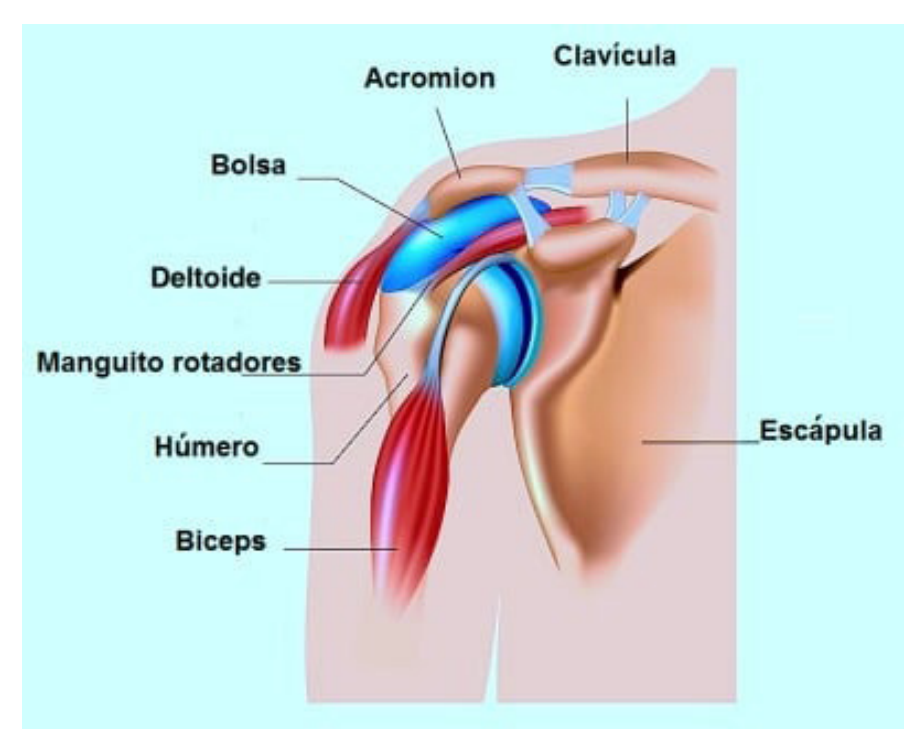

Figura 1. Capsulitis adhesiva o hombro congelado

Fuente: Defilippo, M., (2012)

Entre las enfermedades más comunes del hombro se encuentra el hombro congelado, también conocido como capsulitis adhesiva, que "ocurre cuando los ligamentos que sostienen en el sitio a la articulación del hombro se tensionan debido a una inflamación y restringen el movimiento de la articulación". (Theimer, 2018)

"La cápsula de la articulación del hombro está compuesta de tejido fuerte (ligamentos) que sostiene los huesos del hombro entre sí. Cuando la cápsula resulta inflamada, los huesos del hombro no se pueden mover libremente en la articulación." Esta afección es denominada por (Medlineplus, 2018) como hombro congelado.

Esta patología ha recibido múltiples denominaciones a lo largo de los años, tal y como lo reseña el estudio de Serrano \& Abush (2017):

- Fue nombrada «periartritis escapulohumeral» en 1872 por Duplay, y englobaba un espectro heterogéneo de enfermedades del hombro que presentaban rigidez y dolor.

- El primero en usar el término «hombro congelado" fue Codman en 1934, quien describió un cuadro de dolor difuso e insidioso en el área del músculo deltoides, con incapacidad para dormir sobre el lado afectado y restricción de la movilidad.

- En 1945, Neviaser detalló cambios inflamatorios crónicos y fibrosis en la cápsula articular, con adherencia a la cabeza hu- 
meral; denominó "capsulitis adhesiva» a la obliteración del receso axilar y disminución de volumen de la articulación glenohumeral vistos mediante artrografía.

- Otros términos usados para catalogar esta condición son «Capsulitis retráctil», «periartritis del hombro», «rigidez dolorosa del hombro» y «hombro congelado».

La capsulitis adhesiva es una causa frecuente de hombro doloroso. Para González, Et. Al, (2016):

Es un problema médico comúnmente encontrado, con una frecuencia del 7 al 20\% entre la población adulta. También Ilamada capsulitis adhesiva y es una enfermedad que causa dolor severo en hombro. Su incidencia en la población general va del 2 al $5 \%$, es más común en mujeres de 40 a 60 años y es caracterizada por dolor, pérdida de la función y disminución importante de los rangos de movimiento articular.

\section{Síntomas}

Los dos principales síntomas del hombro congelado son dolor e incapacidad funcional, que es la dificultad de hacer movimientos normales del hombro. La capsulitis adhesiva se desarrolla generalmente en tres etapas, estas etapas son referidas por Pinheiro (2019) según sigue:

- Fase dolorosa o inflamatoria: el cuadro de la capsulitis adhesiva se inicia con movimiento progresivo, dolor al movimiento que llega a ser demasiado intenso y causa también pérdida gradual de la capacidad de mover el hombro. Los síntomas empeoran durante semanas y son generalmente peores en la noche. A diferencia de la bursitis y tendinitis, cuyo dolor se asocian a ciertos movimientos del hombro, el dolor de la capsulitis viene con cualquier tipo de movimiento. Esta fase dura de 2 a 9 meses.

- Fase de congelamiento o rigidez: des- pués de pasada la etapa inflamatoria el dolor comienza a disminuir. Sin embargo, la rigidez del hombro se vuelve más intensa, lo que dificulta su movilidad. En esta etapa, que dura de 4 a 12 meses, la incapacidad funcional no está directamente relacionada con el dolor, el paciente simplemente no puede mover su hombro como antes porque él se encuentra duro o «congelado». En esta etapa, el dolor generalmente ocurre cuando el paciente intenta mover el hombro más allá de lo posible.

- Fase de recuperación o descongelado: después de más de 1 año de dolor e incapacidad funcional, el hombro comienza a «descongelar». El paciente poco a poco va recuperando la capacidad para mover los hombros de forma amplia y el dolor desaparece totalmente. Esta fase puede llevar de 5 a 24 meses para ser completa.

El tiempo de evolución de la enfermedad varía de caso a caso, pero es muy común que el hombro congelado interfiera en las actividades normales de la vida del paciente durante al menos 2 años. Algunos pacientes pueden permanecer con secuelas, perdiendo definitivamente cerca de un 15\% de la movilidad del hombro.

En consonancia con lo indicado por Pinheiro (2019), se hace referencia a estudio publicado por (Mayoclinic, 2018):

El hombro rígido generalmente se manifiesta lentamente y en tres etapas. Cada etapa puede durar meses. Estas son:

- Etapa de bloqueo motor. Cualquier movimiento del hombro genera dolor y la amplitud de movimiento de esta parte del cuerpo comienza a limitarse.

- Etapa rígida. El dolor puede comenzar a disminuir durante esta etapa. Sin embargo, el hombro se torna rígido y es cada vez más difícil usarlo.

- Etapa de descongelamiento. La ampli-

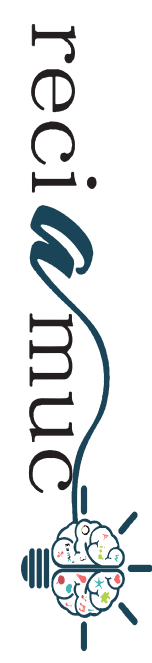




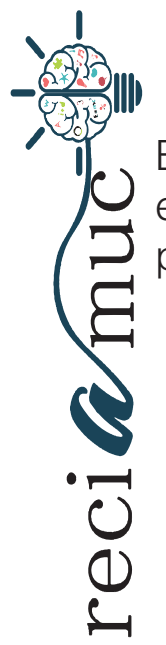

tud de movimiento del hombro comienza a mejorar.

El dolor que produce el hombro congelado es generalmente un dolor fijo o continuo. Típicamente es peor en la primera etapa del

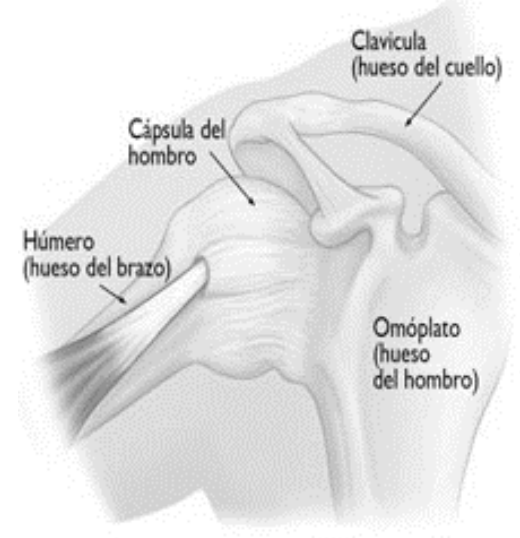

curso de la enfermedad. El dolor por lo general se localiza en el área exterior del hombro y en ocasiones puede afectar el brazo (la parte que va del codo al hombro).

Figura 2. Hombro congelado

Fuente: Fisioonline, (2018).

\section{Diagnóstico}

El diagnóstico de hombro congelado generalmente se obtiene tras la realización de una historia clínica, lo que marcará el diagnóstico diferencial de la capsulitis adhesiva. En este sentido Monreal, Et. Al (2006) describen que:

El diagnóstico del hombro congelado se basa en la historia y el examen físico. Por definición es una condición idiopática, por tanto, se realiza por exclusión. Aunque muchas condiciones pueden precipitar su aparición, debe descartarse la ruptura del manguito rotador, las fracturas, luxaciones no diagnosticadas, tendinitis calcificada, Condrocalcinosis, las parálisis, tumores locales o metastásicos, el dolor irradiado o histérico, necrosis Avascular u otra enfermedad ósea.

Según la presunción diagnóstica se solicitan exámenes imagenológicos, principalmente para descartar otras patologías. Entre los exámenes usualmente utilizados (Orthoinfo,
2014) destaca los siguientes:

- Rayos X. Las estructuras densas, como el hueso, se ven claramente con los rayos $X$. Los rayos $X$ podrían mostrar otros problemas en su hombro, por ejemplo osteoartritis.

- Imagenología de resonancia magnética (MRI) y ultrasonido. Estos estudios pueden crear mejores imágenes de los problemas con tejidos blandos, por ejemplo un manguito rotador desgarrado. 


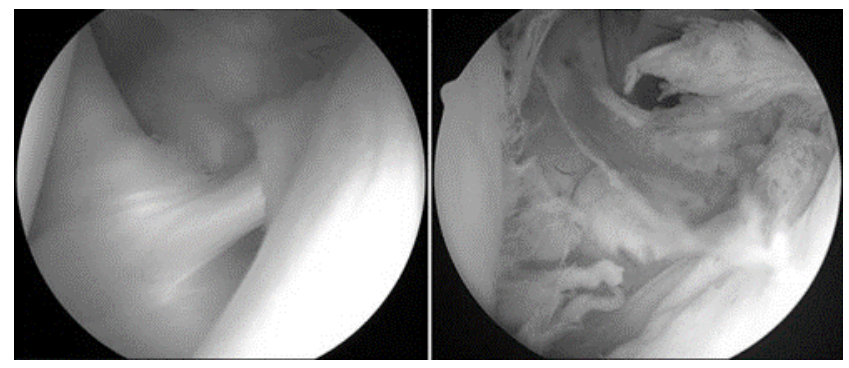

Figura 3. Imagenología hombro congelado

Fuente: Fisioonline, (2018).

Tratamiento

El tratamiento indicado para esta patología va a depender de que tan avanzada se encuentre la enfermedad y el mismo debe ser individualizado. Al respecto Tlatoa, Et. Al (2014) destacan los siguientes tipos de tratamiento:

- Opciones farmacológicas, los antiinflamatorios no esteroideos (AINE) no tienen evidencia bibliográfica de efectividad ya que nunca han mostrado mejora funcional ni de dolor en relación al placebo. Por parte de los corticoides han demostrado disminuir el dolor e incrementar el rango de movimiento hasta 6 semanas, pero al mismo tiempo existe los riesgos propios de este tipo de medicamentos (rotura tendinosa, necrosis grasa, y glicemia). Mediante Infiltración subacromial se han obtenido mejores resultados que vía oral y se considera una técnica segura y efectiva.

- Fisioterapia con ejercicios de rango de movimiento a tolerancia progresivos y estiramientos pasivos. La opción más tradicional corresponde a ejercicios con peso y movimientos pendulares.

- Hidrodilatación y movilización bajo anestesia. La primera consiste en generar una dilatación de la cápsula articular mediante suero salino bajo ultrasonido, en ocasiones se agregan corticoides; esto genera una distención y ruptura de la cápsula con mejoras sintomáticas de 6 a 12 semanas. La segunda está in- dicada a los 6meses de fracaso en el tratamiento, en donde se anestesia al paciente y se realizan movilizaciones del hombro en todos los planos hasta escuchar el desgarro de la cápsula. Hasta ahora la hidrodilatación presenta mejores resultados que la movilización bajo anestesia, la cual requiere de mayor evidencia.

- La opción quirúrgica, se considera a las 6 a 12 semanas de un tratamiento conservador fallido. La técnica más utilizada es una liberación capsular artroscópica, por su parte la técnica abierta es rara, ya que puede generar bridas.

Al respecto, Arce, Et. Al (2008) indican que:

Sin importar la etiología, el tratamiento inicial es siempre conservador, con terapia física, ejercicios de elongación, antiinflamatorios no esteroides e infiltraciones con corticoides. Con este tratamiento se observa un porcentaje variable de fracasos $(7 \%$ al $10 \%$ ). En esos casos se consideran los distintos tratamientos quirúrgicos, entre los que se encuentran la manipulación bajo anestesia, la manipulación con el agregado de desbridamiento artroscópico18 con descompresión subacromial o no, y la capsulotomía selectiva artroscópica. Estas capsulotomías se basan en la liberación de las cápsulas anterior y posterior para recuperar la rotación externa e interna respectivamente.

En relación al tratamiento de esta patología los profesionales de la salud deben hacer 
énfasis en educar al paciente en cuanto a las fases de ésta enfermedad y dejar claro que el rango de movimiento mejora, pero puede no volver a la normalidad.

\section{Conclusiones}

El Hombro Congelado es una pérdida gradual del movimiento que se acompaña de dolor difuso y donde varios autores han descrito posibles causas y cambios patológicos que ocurren en esta importante zona y en las partes que la constituyen internamente.

Por lo general, los signos y síntomas comienzan de forma gradual, empeoran con el tiempo y luego se resuelven, generalmente en un plazo de uno a tres años. Algunos pacientes pueden permanecer con secuelas, perdiendo definitivamente cerca de un $15 \%$ de la movilidad del hombro.

El tratamiento del hombro rígido comprende ejercicios de amplitud de movimiento y, medicamentos antiinflamatorios no esteroides e infiltraciones con corticoides que se inyectan en la cápsula articular. En un pequeño porcentaje de los casos, se puede indicar una cirugía artroscópica para aflojar la cápsula articular y que pueda moverse con más libertad.

El consenso mayoritario con respecto a la capsulitis adhesiva es que es muy desconocida, en particular en lo que se refiere a su etiología, lo que obliga a los profesionales de la salud a realizar una historia clínica detallada del paciente a fin de poder emitir un diagnostico certero y posterior aplicación de tratamiento que permita mejorar los síntomas de esta patología que afecta significativamente la calidad de vida del paciente.

\section{Bibliografía}

Serrano, A., \& Abush, S. (03 de 2017). Capsulitis adhesiva. Anales Medicos, 62(1), 37-43. Recuperado el 08 de 01 de 2020, de https://www.medigraphic.com/pdfs/abc/bc-2017/bc171h.pdf

Tlatoa, H., Morales, F., \& Ocaña, H. (2014). Actuali- zación en Traumatología Deportiva: hombro congelado. Elsevier, 22, 132/137. doi:DOI: 10.1016/ S2214-3106(15)30010-8

Arce, G., Lacroze, P., Previgliano, J., Schamis, O., Pereira, E., \& Arcuri, F. (28 de 03 de 2008). Tratamiento quirúrgico de la capsulitis. Rev Asoc Argent Ortop Traumatol, 73(1), 13-19.

González, B., Chávez , G., \& Lara, E. (12 de 2016). Hombro Congelado. Reporte de caso exitoso con rehabilitación. Conamed, 21(4), 192-196. Recuperado el 07 de 01 de 2020, de https://www.medigraphic.com/pdfs/conamed/con-2016/con164f.pdf

Healthwise. (26 de 06 de 2019). northshore Health Encyclopedia. Recuperado el 09 de 01 de 2020, de https://www.northshore.org/healthresources/ encyclopedia/encyclopedia.aspx?DocumentHwi$\mathrm{d}=$ tw9148spec\&Lang=es-us

Mayoclinic. (20 de 07 de 2018). Mayoclinic. Recuperado el 07 de 01 de 2020, de https://www.mayoclinic.org/es-es/diseases-conditions/frozen-shoulder/symptoms-causes/syc-20372684

Medlineplus. (04 de 09 de 2018). Medlineplus. Recuperado el 07 de 01 de 2020, de https://medlineplus.gov/spanish/ency/patientinstructions/000550. htm

Monreal, R., Díaz, H., León, P., \& Paredes, L. (12 de 2006). Capsulitis adhesiva del hombro: tratamiento con distensión hidráulica y anestesia local. Revista Cubana de Ortopedia y Traumatología, 20(2). Recuperado el 08 de 01 de 2020, de http:// scielo.sld.cu/scielo.php?script=sci_arttext\&pi$d=$ S0864-215X2006000200005

Orthoinfo. (01 de 2014). Orthoinfo. Recuperado el 08 de 01 de 2020, de https://orthoinfo.aaos. org/es/diseases--conditions/hombro-congelado-frozen-shoulder/

Ortiz, L., Larrosa, H., \& De Miguel, E. (2010). Capsulitis adhesiva del hombro: una revisio'n sistema'tica. Fisioterapia, 32(5), 229-235.

Pinheiro, P. (31 de 07 de 2019). mdsaude. Recuperado el 08 de 01 de 2020, de https://www.mdsaude.com/es/ortopedia-es/capsulitis-adhesiva-hombro-congelado/

Ramos, J. (2017). TERAPIA MANUAL ORTOPÉDICA EN. Trabajo de Suficiencia Profesional, Universidad Inca Garcilaso De La Vega, Lima.

Serrano, A., \& Abush, S. (03 de 2017). Capsulitis adhesiva. Anales Medicos. Asociacion Medica Centro Medico ABC, 62(1), 37-43. Recuperado el 08 de 01 de 2020, de https://www.medigraphic.com/ pdfs/abc/bc-2017/bc171h.pdf 
Theimer, S. (06 de 06 de 2018). Mayoclinic. Recuperado el 08 de 01 de 2020, de https://newsnetwork. mayoclinic.org/discussion/preguntas-y-respuestas-tratamiento-para-el-hombro-congelado/

Ugalde, C., Zúñiga, D., \& Barrantes, R. (03 de 2013). Actualización del síndrome de hombro doloroso: lesiones del manguito rotador. Medicina legal de costa rica, 30(1). Recuperado el 07 de 01 de 2020, de http://www.scielo.sa.cr/scielo.php?script=sci_ arttext\&pid=S1409-00152013000100009

\section{CITAR ESTE ARTICULO:}

Versoza Castro, K., Zambrano Sanchez, C., Masache Galvez , L., \& Sanchez Ortiz, B. (2020). Hombro congelado. Diagnóstico y tratamiento. RECIAMUC, 4(1), 267-275. doi:10.26820/reciamuc/4.(1).enero.2020.267-275

\section{(c) $(1) \odot$ BY NC SA}

RECONOCIMIENTO-NOCOMERCIAL-COMPARTIRIGUAL CC BY-NC-SA

ESTA LICENCIA PERMITE A OTROS ENTREMEZCLAR, AJUSTAR Y CONSTRUIR A PARTIR DE SU OBRA CON FINES NO COMERCIALES, SIEMPRE Y CUANDO LE RECONOZCAN LA AUTORÍA Y SUS NUEVAS CREACIONES ESTÉN BAJO UNA LICENCIA CON LOS MISMOS TÉRMINOS. 